\title{
Design and Analysis of Non-Uniformly Spaced Dipole Array Antenna
}

\author{
N Soujanya ${ }^{1}$, Dr. Mahesh $\mathrm{A}^{2}$ \\ ${ }^{1} P G$ student, Department of Electronics and Communication Engineering \\ ${ }^{2}$ Associate Professor, Department of Electronics and Communication \\ $R V$ College of Engineering, Bengaluru, India \\ ${ }^{1}$ Email: nsoujanya.lcs19@rvce.edu.in \\ 2Email: mahesha@rvce.edu.in
}

\begin{abstract}
Dipole antenna array is designed at operating frequency of $450 \mathrm{MHz}$ using FR4 substrate. Uniform circularly polarized (UCP) and non-uniform circularly polarized (NUCP) dipole arrays are designed. NUCP array is achieved by placing antenna elements at unequal distance from the reference element. Low side lobe level is necessary to reduce interference with other frequencies in the band which is achieved in case of NUCP array compared UCP array. The simulation is carried out using EM flow solver HFSS. The simulation results indicate that there is a reduction in the side lobe level for the $1 x 7$ NUCP array as compared to UCP array. To enhance the gain of the dipole array dielectric lens is used as the secondary radiator which also acts as a radome to protect the array. The maximum gain achieved is $1.59 d B$ with lens. Return loss less than -15dB is achieved in all cases. Axial ratio less than $3 d B$ achieved for circular polarized arrays. The designed NUCP array with lens can be used in SAR (synthetic aperture radar) applications.
\end{abstract}

Keywords: UCP, NUCP, Circular polarization, Axial ratio, Return loss, SAR.

\section{INTRODUCTION}

In the area of communication systems, whenever the requirement of wireless communication arises, there occurs the necessity of an antenna. Antenna is the connection between radio waves propagating through space and electric currents propagating through transmission lines. In order to improve the performance of the system, antenna arrays are designed. Dipole antenna is most widely used antenna because of its small size, ease of construction and high power efficiency. One of the factors that need to be considered when designing an antenna is polarization. Antennas are sensitive to the polarization if the polarization of antenna does not match the signal, then the signal level decreases correspondingly. Circular polarization eliminates the need to align antenna modules.

Uniform linear arrays exhibit high side lobe level in the radiation pattern which causes interference of the signals which degrades the performance of the array. One of the methods to reduce side lobe levels in Circular Polarized array is by using non-uniform configuration which reduces the side lobe level compared to uniform configuration. In order to enhance the gain of the antenna arrays, hemispheroidal dielectric lens antenna is employed. Dielectric lenses are easy to fabricate, cost effective, have low dimensional tolerance and are suitable for wide frequency ranges, microwave frequency and steering applications.

\section{CP \& NUCP DIPOLE ANTENNA ARRAY DESIGN}

The dipole antenna structure is designed at $450 \mathrm{MHz}$ resonant frequency using FR4 substrate of thickness $1.6 \mathrm{~mm}$ with dielectric constant of 4.4 and loss tangent of 0.02 is shown in Figure 1. The simulated HFSS results of Return loss $=-21.98 \mathrm{~dB}$, Gain $=2.19 \mathrm{~dB}$ and Axial ratio $=96.72 \mathrm{~dB}$ is obtained as shown in Figure 2, 3 and 4 respectively. 


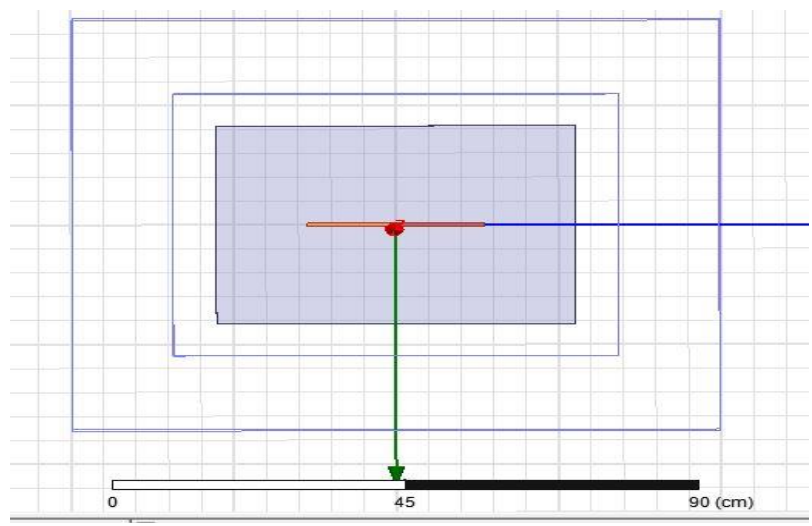

Figure 1. Dipole antenna

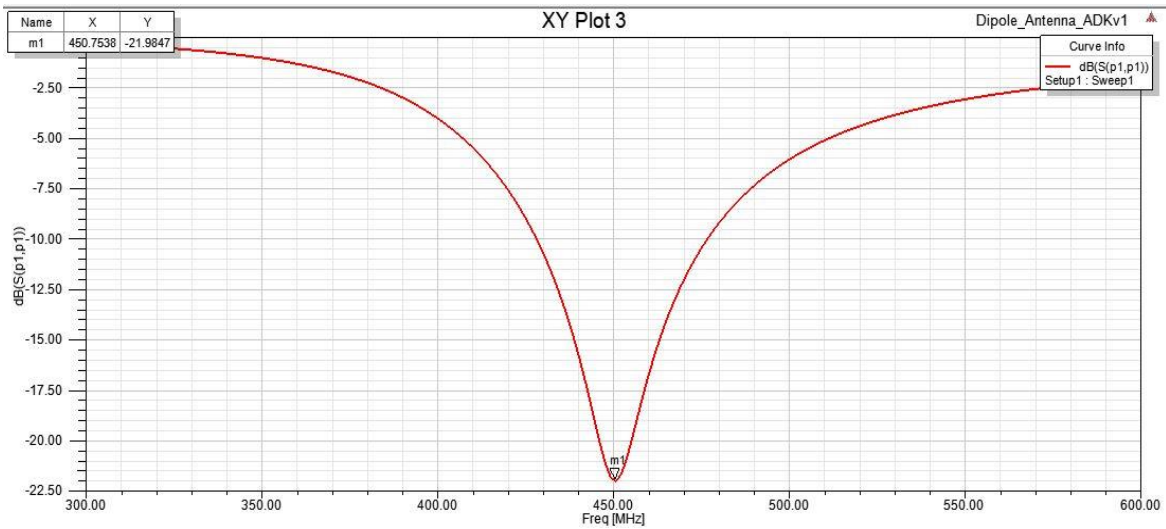

Figure 2. Return loss of dipole antenna

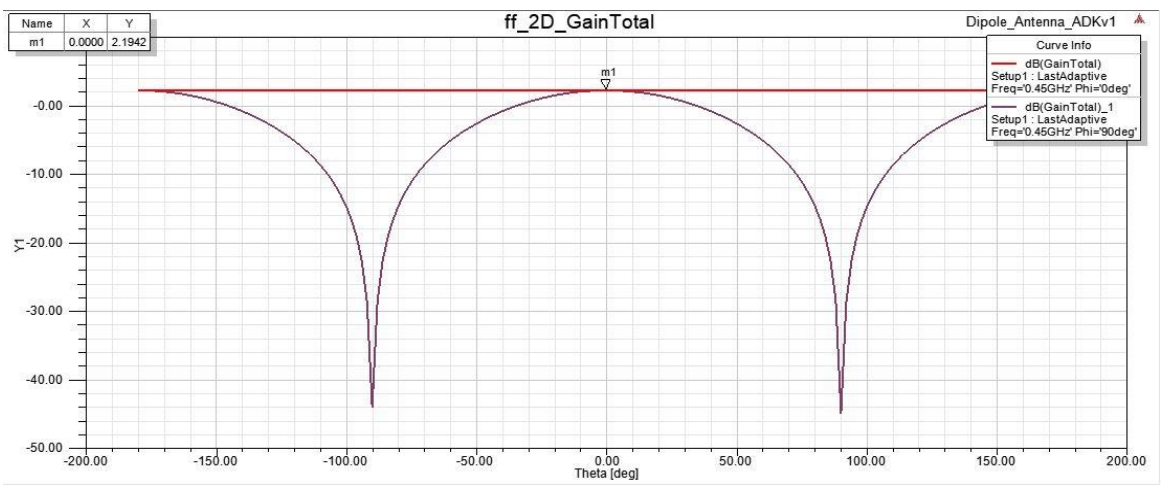

Figure 3. Radiation pattern of dipole antenna

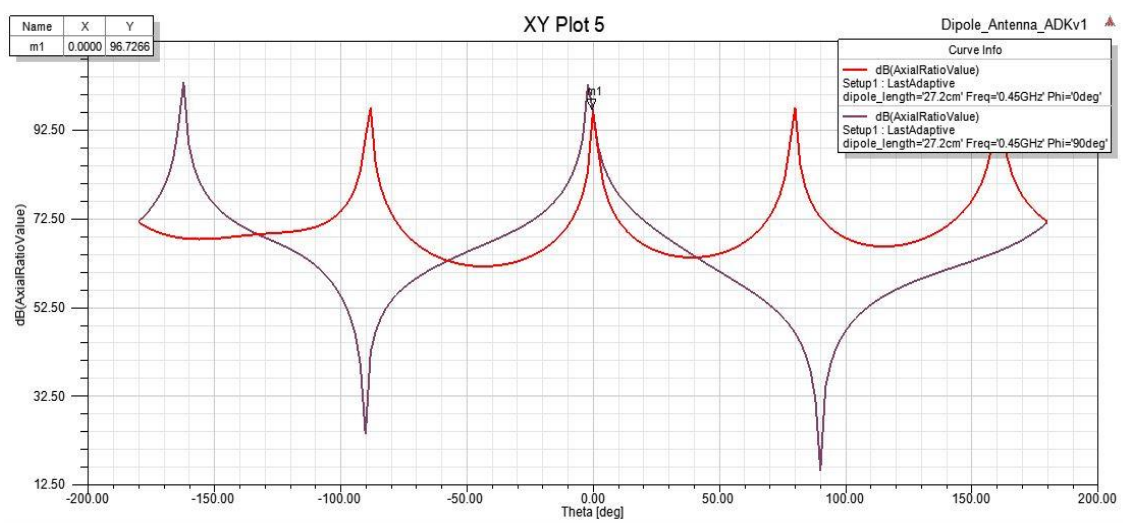

Figure 4. Axial ratio of dipole antenna 


\subsection{1x4 UCP and NUCP Dipole Array Design}

Dipole antenna operates in linear polarization. $\mathrm{CP}$ is achieved by arranging four dipoles on same substrate which is referred as unit cell. 1x4 UCP array is designed by placing unit cells at a distance $d=\lambda / 7$ is shown in Figure 5 . Return loss of $-15.79 \mathrm{~dB}$ return loss, gain of $11.32 \mathrm{~dB}$ and axial ratio of $0.54 \mathrm{~dB}$ is obtained. $1 \times 4 \mathrm{NUCP}$ is designed by placing unit cells at a distance of $d, 2 d$ and $3 \mathrm{~d}$ as shown in Figure 6. Return loss of $-15.76 \mathrm{~dB}$, Gain value of $11.81 \mathrm{~dB}$ and AR of $0.37 \mathrm{~dB}$ is obtained. Normalized radiation pattern of UCP and NUCP array is shown in Figure 7. The SLL for UCP array is $-13.69 \mathrm{~dB}$ and for NUCP array is $-12.89 \mathrm{~dB}$. It is observed that the SLL for NUCP array is more than UCP array.

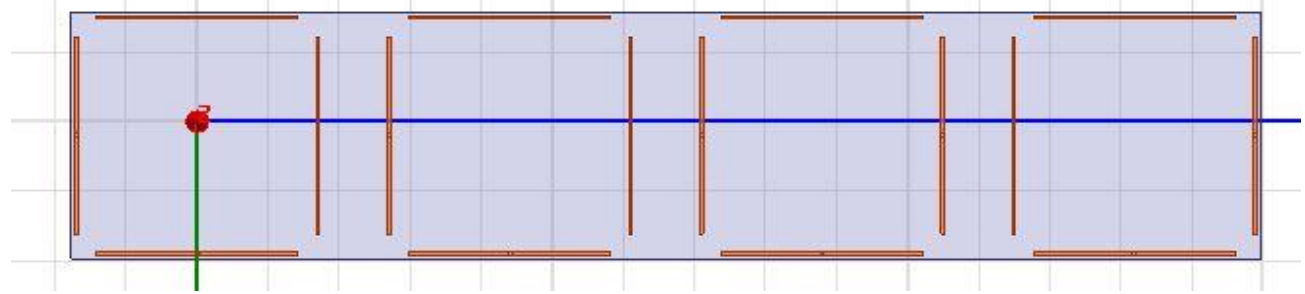

Figure 5. 1x4 UCP dipole array

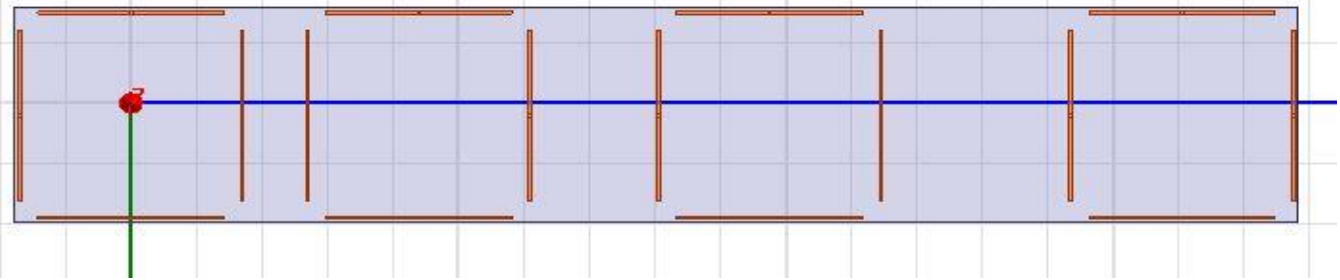

Figure 6. 1x4 NUCP dipole array

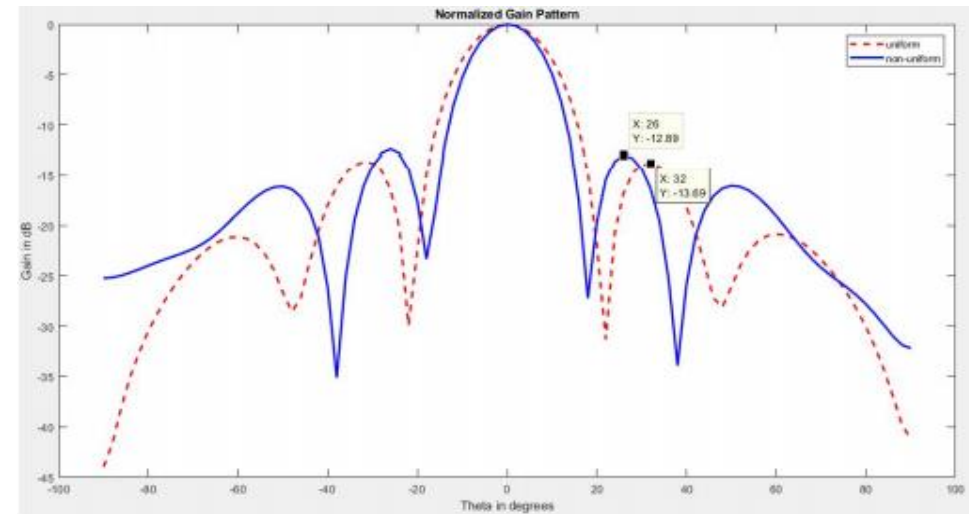

Figure 7. Normalized radiation pattern of 1x4 UCP and NUCP dipole array

\subsection{1x7 UCP and NUCP Dipole Array Design}

$1 \mathrm{x} 7 \mathrm{UCP}$ array is designed by placing unit cells at a distance $\mathrm{d}=\lambda / 7$ is shown in Figure 8 . Return loss of $-16.19 \mathrm{~dB}$ return loss, gain of $13.48 \mathrm{~dB}$ and axial ratio of $0.60 \mathrm{~dB}$ is obtained. 1x7 NUCP is designed by placing unit cells at a distance of $\mathrm{d}, 2 \mathrm{~d}$ and $3 \mathrm{~d}$ as shown in Figure 9. Return loss of $-16.21 \mathrm{~dB}$, Gain value of $13.97 \mathrm{~dB}$ and AR of $0.57 \mathrm{~dB}$ is obtained. Normalized radiation pattern of UCP and NUCP array is shown in Figure 10. The SLL for UCP array is $-13.54 \mathrm{~dB}$ and for NUCP array is $-15.18 \mathrm{~dB}$. It is observed that the SLL for NUCP array is less than UCP array.

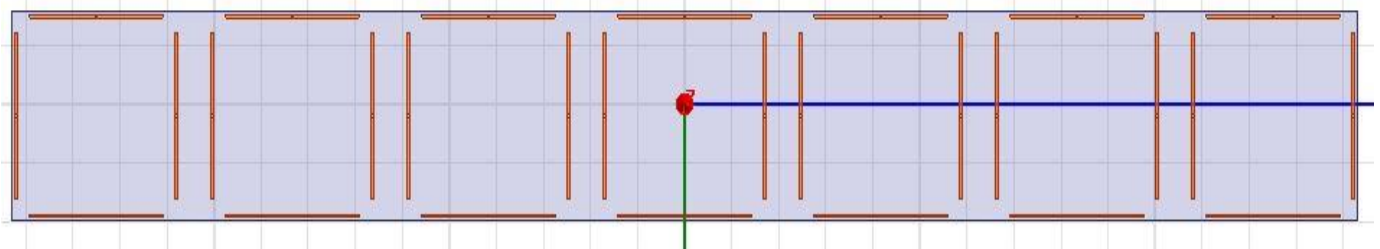

Figure 8. 1x7 UCP dipole array 


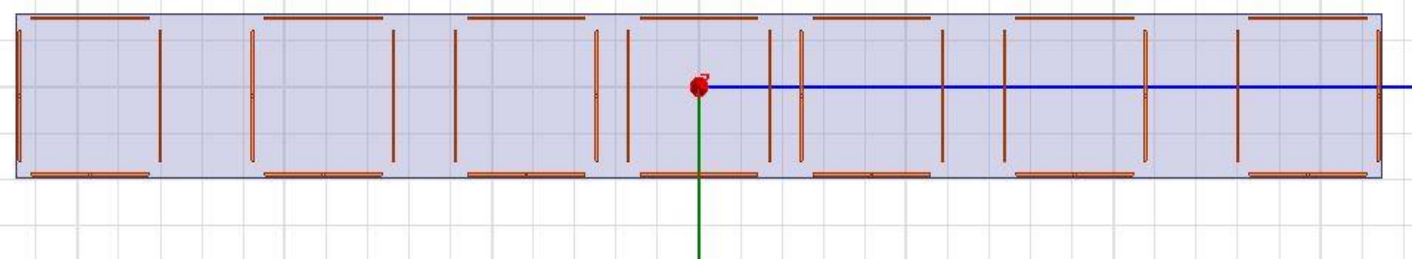

Figure 9. 1x7 NUCP dipole array

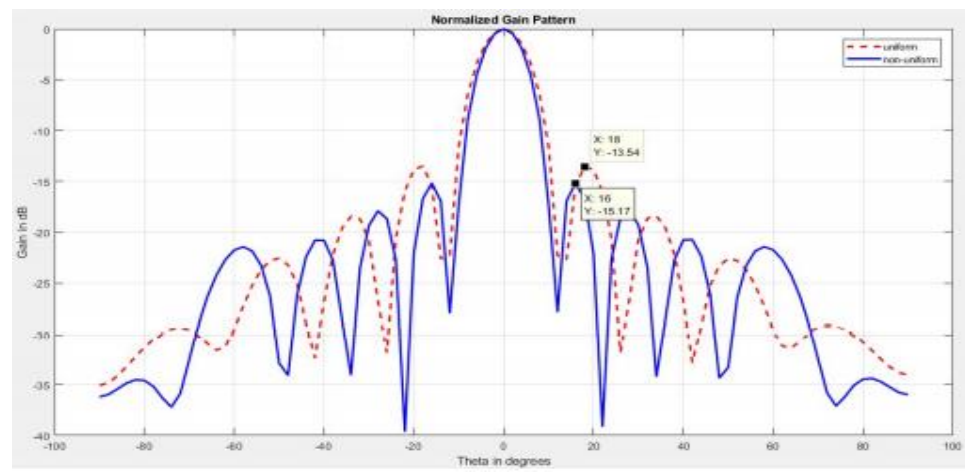

Figure 10. Normalized radiation pattern of 1x7 UCP and NUCP dipole array

\subsection{1x4 UCP and NUCP array with lens}

$1 \mathrm{x} 4 \mathrm{UCP}$ array is designed along with hemispheroidal lens of polypropylene material with dielectric constant 2.2 and loss tangent 0.002 as shown in Figure 11. The dimensions of lens is $320 \mathrm{~cm} \times 80 \mathrm{~cm}$ (Major axis $\mathrm{x}$ Minor axis) placed at an optimized distance of $4 \mathrm{~cm}$ from array. $1 \times 4$ NUCP array along with lens is shown in Figure 12. The dimension of lens is $400 \mathrm{~cm} \times 100 \mathrm{~cm}$ placed at an optimized distance of $10 \mathrm{~cm}$. The gain enhancement of $1.52 \mathrm{~dB}$ and $1.59 \mathrm{~dB}$ is respectively observed in $1 \mathrm{x} 4 \mathrm{UCP}$ and NUCP array with lens as shown in Figure 13 and 14.

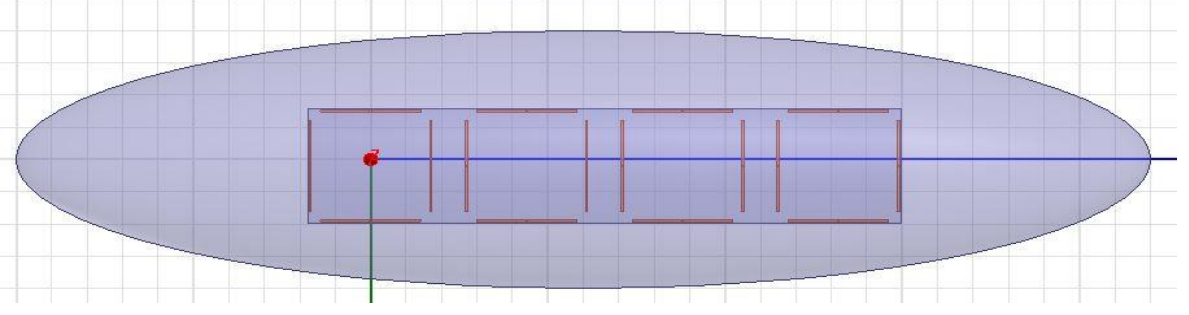

Figure 11. 1x4 UCP array with lens

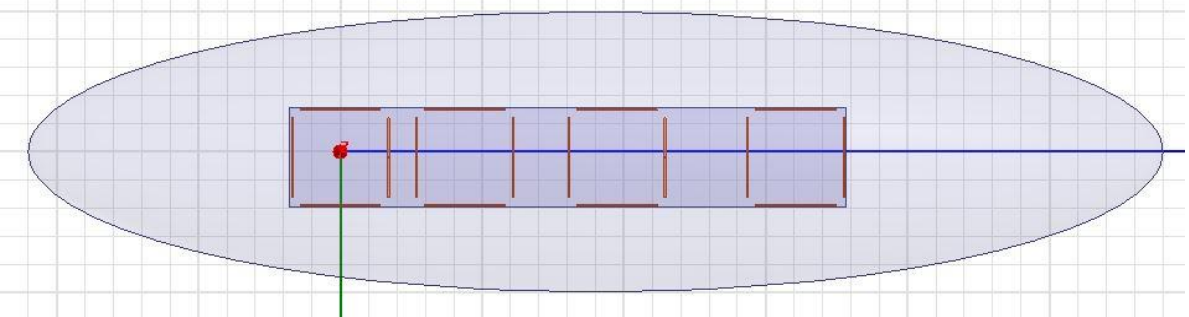

Figure 12. 1x4 NUCP array with lens

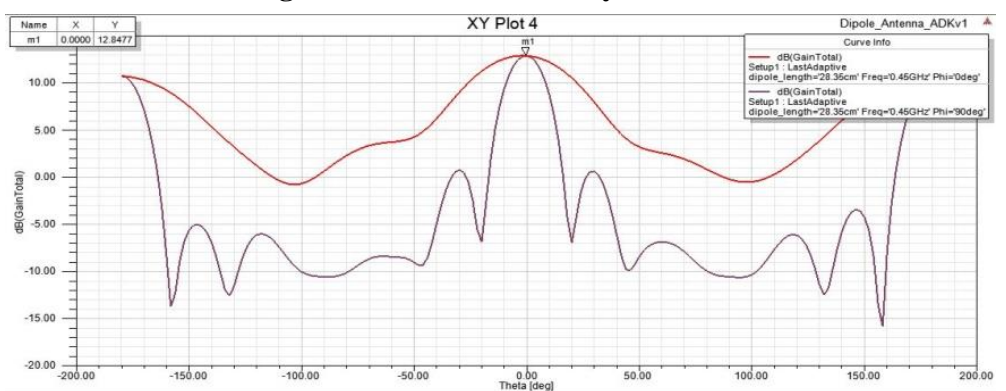

Figure 13. Gain of 1x4 UCP array with lens 


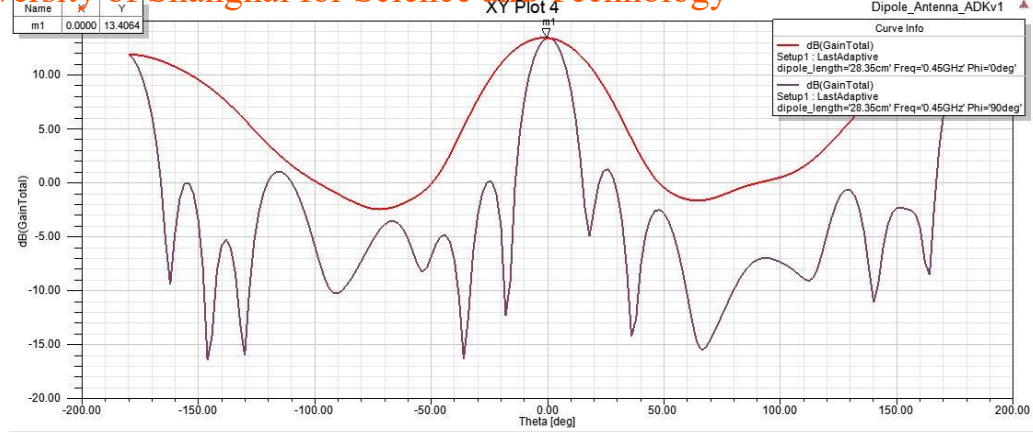

Figure 14. Gain of 1x5 NUCP array with lens

\subsection{1x7 UCP an NUCP array with lens}

1x7 UCP array is designed along with hemispheroidal dielectric lens as shown in Figure 15. The dimensions of lens is $400 \mathrm{~cm} \times 100 \mathrm{~cm}$ (Major axis $\mathrm{x}$ Minor axis) placed at an optimized distance of $4 \mathrm{~cm}$ from array. $1 \times 7$ NUCP array along with lens is shown in Figure 16. The dimension of lens is $500 \mathrm{~cm} \times 100 \mathrm{~cm}$ placed at an optimized distance of $10 \mathrm{~cm}$. The gain enhancement of $1.04 \mathrm{~dB}$ and $1.5 \mathrm{~dB}$ is respectively observed in $1 \mathrm{x} 4 \mathrm{UCP}$ and NUCP array with lens as shown in Figure 17 and 18.

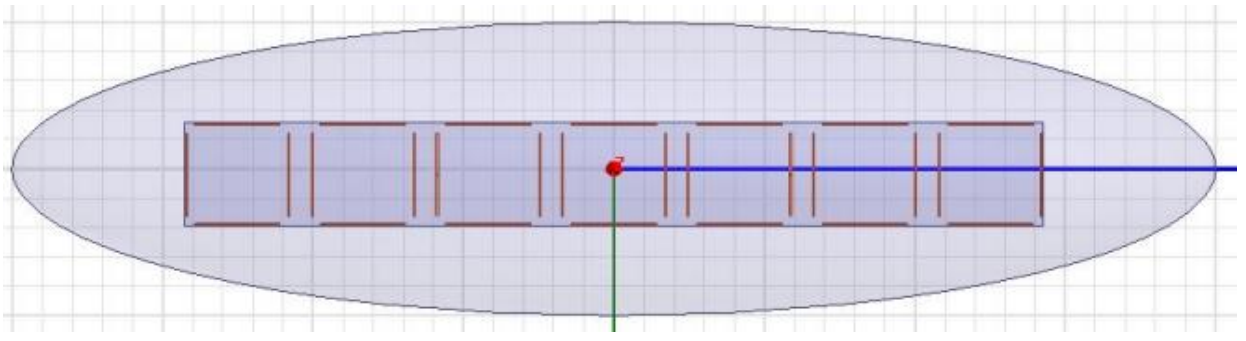

Figure 15. 1x7 UCP array with lens

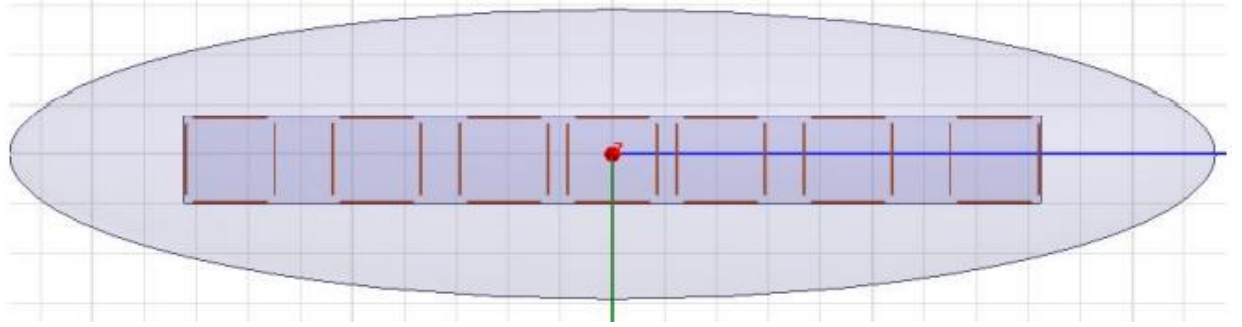

Figure 16. 1x7 NUCP array with lens

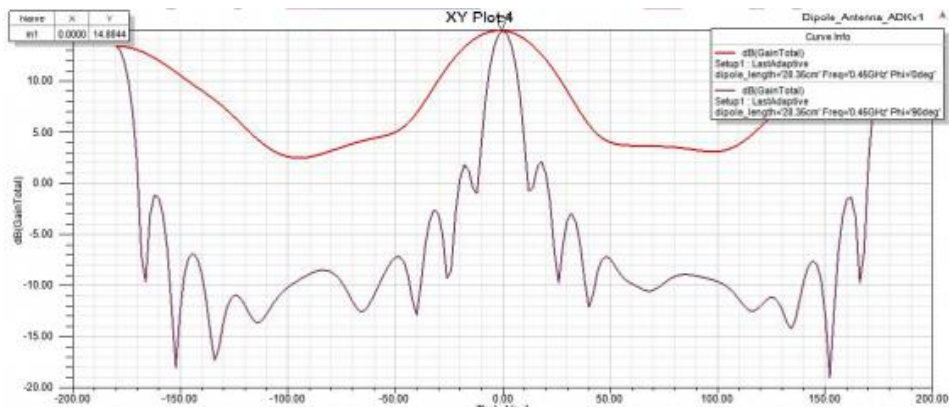

Figure 17. Gain of $1 \times 7$ UCP array with lens

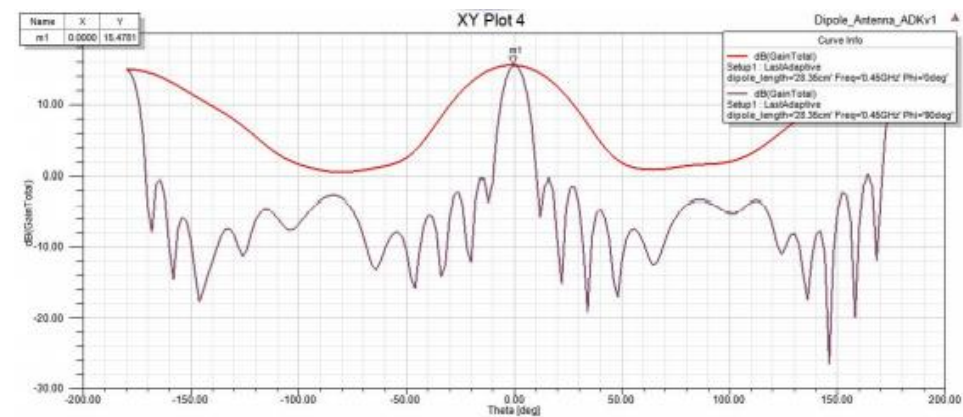

Figure 18. Gain of $1 \times 7$ NUCP array with lens 
The results for gain with and without lens for all cases are tabulated in Table 1.The maximum gain enhancement of $1.59 \mathrm{~dB}$ is observed with lens in case of $1 \mathrm{x} 4 \mathrm{NUCP}$ array. The beam steering employed for the designed dipole array for SAR applications. The Figure 18, 19, 20 and 21 respectively shows beam steering plots for 1x7 UCP, NUCP, UCP with lens and NUCP with lens. It is observed from the simulation results, the maximum scan angle possible is $\pm 30^{\circ}$ without deteriorating the radiation pattern and gain.

Table 1. Comparison of gain without and with lens

\begin{tabular}{|l|c|c|}
\hline Array structures & $\begin{array}{c}\text { Gain without } \\
\text { lens }(\mathrm{dB})\end{array}$ & $\begin{array}{c}\text { Gain with } \\
\text { lens }(\mathrm{dB})\end{array}$ \\
\hline $1 \times 4$ UCP array & 11.32 & 12.84 \\
\hline $1 \times 4$ NUCP array & 11.81 & 13.40 \\
\hline $1 \times 7$ UCP array & 13.48 & 14.88 \\
\hline $1 \times 7$ NUCP array & 13.97 & 15.47 \\
\hline
\end{tabular}
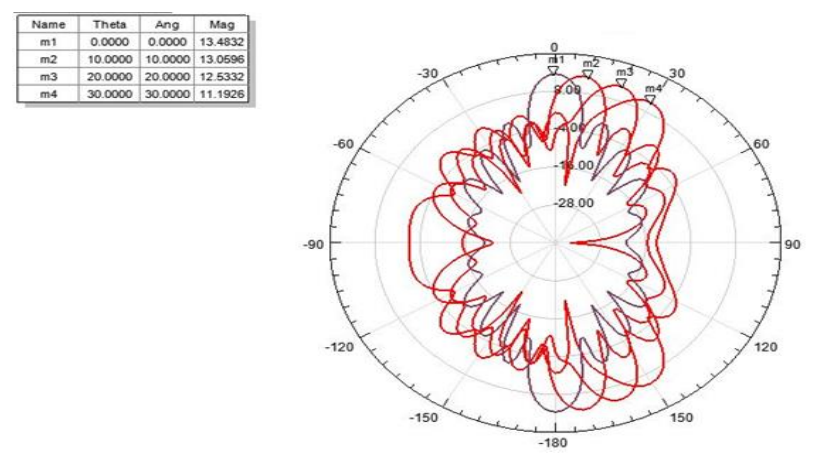

Figure 18. Beam steering of 1x7 UCP array
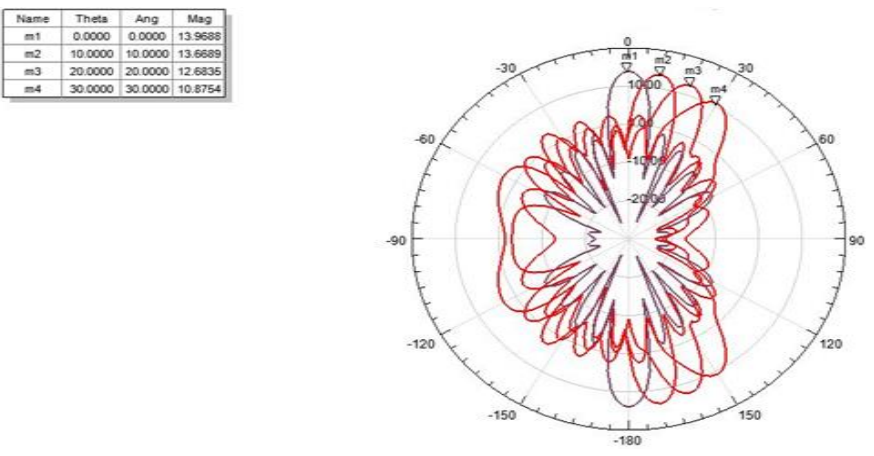

Figure 19. Beam steering of 1x7 NUCP array

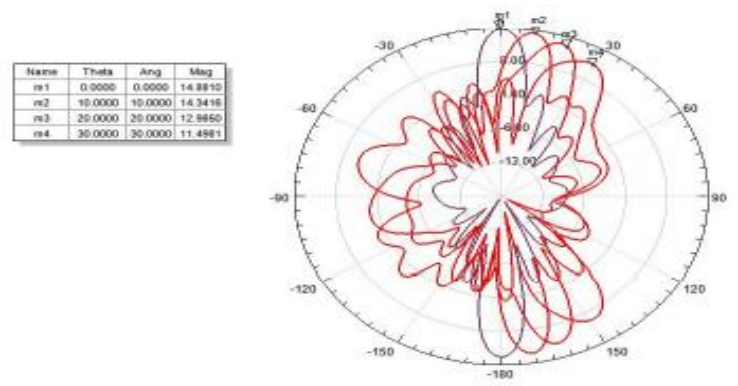

Figure 20. Beam steering of 1x7 UCP array with lens 

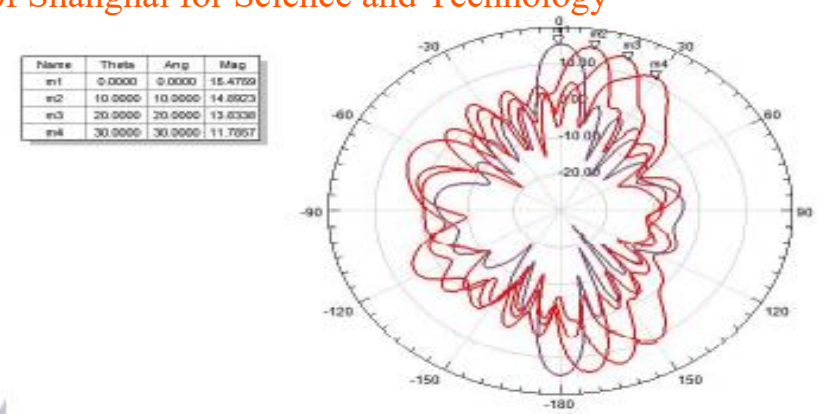

Figure 21. Beam steering of 1x7 NUCP array with lens

\section{CONCLUSION}

The design of $1 \times 4$ and $1 \times 7$ UCP and NUCP dipole arrays is presented. The arrays are designed and simulated using HFSS software. From simulation results it is observed that the sidelobe level of $1 \times 7$ NUCP array is less compared to $1 \times 4$ NUCP array. The array gain is enhanced by using a dielectric lens of hemispheroidal shape. The low loss dielectric material Polypropylene is used for the lens. The maximum gain enhancement of $1.59 \mathrm{~dB}$ is observed with lens in case of $1 \times 4$ NUCP array. The arrays have the capability to scan the beam till $\pm 30^{\circ}$ by retaining the shape of the pattern with minimal gain reduction. The same effect is observed with lens also. The designed NUCP array with lens is used for SAR applications.

\section{ACKNOWLEDGEMENT}

The authors would like to thank RV College of engineering for providing the simulation resources to carry out the research work.

\section{REFERENCES}

[1] Denis Letavin, PolinaZiomkovskaia, "Microstrip Dipole Antenna with Circular Po larization of Four Identical Dipole", IEEE Radiation and Scattering of Electromagnetic Waves, Divnomorskoe, Russia, 2019.

[2] AsifJamilSahad, Md. Shazzadul Islam, Md. Rafiqul Islam, G. M. Asadullah, Noor Hidayah M Adnan, "Design and Analysis of a Non-Uniform Planar Antenna Array",IEEE Region 10 Symposium (TENSYMP), Dhaka, Bangladesh, 2020.

[3] ShirzadShahriari, Yoon Choi, Amir Eshraghi, H. K. Hwang, ZekeriyaAliyazicioglu, “Antenna Beam Shaping Using a Non-Uniform Array Antenna”, IEEE 4th International Conference on Recent Advances in Space Technologies, Istanbul, Turkey, 2009.

[4] Son Xuat Ta and Ikmo Park, "Wideband Circularly Polarized Dipole Array Using a Sequential-Phase Feed", IEEE International Symposium on Antennas and Propagation, Fajardo, PR, USA, 2016.

[5] EhsanTohidi, Mohammad A, Mohammad M. Nayebi, and Hamid Behroozi, "A fast and novel method of pattern synthesis for non-uniform phased array antennas", IEEE 16th International Radar Symposium,Dresden, Germany, 2015.

[6] M. Laribi, N. Hakem, "Gain-Bandwidth Enhancement of $60 \mathrm{GHz}$ DRA Using Lens Technique," IEEE International Symposium on Antennas and Propagation, Fajardo, PR, USA, 2016. 
[7] Mahesh A, Ravishankar S, Rukmini T S, Thakur S K, "The Gain Enhancement of Microstrip Array Antenna with Dielectric Lens-A Comparative Study”, IEEE Applied Electromagnetics Conference, Kolkata, India, 2009.

[8] Wenfei Yin, Hongzhi Zhao, Zhaoneng Jiang, "Gain-Enhanced Broadband Circularly Polarized Crossed Dipole Antenna”, IEEE International Conference on Computational Electromagnetics, Shanghai, China, 2019.

[9] MuradRidwan, Mohammed Abdo, Eduard Jorswieck, "Design of Non-Uniform An tenna Arrays Using Genetic Algorithm”, IEEE 13th International Conference on Ad vanced Communication Technology, Gangwon, Korea, 2011. 